Johannes F. K. Schmidt

\title{
Beziehung als systemtheoretischer Begriff
}

Zusammenfassung: Den Begriff der sozialen Beziehung kann man sozialtheoretisch, aber auch differenzierungstheoretisch verstehen. Hinsichtlich der erstgenannten Lesart - Sozialität als Beziehung zwischen Menschen - findet man bei Luhmann eine polemische Ablehnung, während er die zweite Lesart - Beziehung als eine spezifische soziale Form - in einer theoretisch weitgehend unkontrollierten Art verwendet und eine Abstimmung mit dem Theorem der sozialen Differenzierung (Interaktion, Organisation, Gesellschaft) nicht vorgenommen hat. Es ist aber gerade die Luhmannsche Lesart des Interaktionsbegriffs in der Nachfolge Goffmans, die die Systemtheorie gegenüber Phänomenen wiederholter Interaktion seltsam sprachlos erscheinen lässt. Deshalb wird hier vorgeschlagen, den Beziehungsbegriff als eine Selbstbeschreibung eines spezifischen sozialen Systems in Form der Interdependenz von Interaktionen zu verstehen.

\section{Einleitung}

In der Luhmannschen Differenzierungstheorie fällt die geringe Zahl der möglichen Formen auf, für die Luhmann $(1997,612)$ ein nicht weiter spezifiziertes Gesetz begrenzter Möglichkeiten unterstellt. Dies gilt nicht nur für die gesellschaftliche Differenzierung mit der Unterscheidung von segmentär, stratifikatorisch und funktional differenziert, sondern auch und besonders für die soziale Differenzierung mit der Unterscheidung von Interaktion, Organisation und Gesellschaft. Es handelt sich bei den jeweils genannten Differenzierungstypologien aber nicht um abgeschlossene Listen. Vielmehr hat Luhmann im Verlauf der Theorieentwicklung eher induktiv und den Realitäten der Gesellschaft geschuldet weitere Systemformen hinzugefügt. Für den Bereich der sozialen Differenzierung nennt er selbst - wenn auch offensichtlich mit einem gewissen Unbehagen $(1997,813)$ und »ohne Rücksicht auf Theorieästhetik « $(1997,847)$ - das Phänomen der Protestbewegungen. Daneben hat es in der Luhmann rezipierenden Literatur immer wieder weitere Vorschläge gegeben, hierbei ist insbesondere der Begriff der Gruppe zu nennen (vgl. Willke 1978; Neidhardt 1979; Tyrell 1983). Dazu hat sich Luhmann selbst nie explizit geäußert. ${ }^{1}$ Eine ausdrückliche und polemische Ablehnung findet man dagegen bei

1 Dies ist insofern überraschend, da der Gruppenbegriff in der frühen Organisationssoziologie von 1964 noch eine wichtige Rolle gespielt hat (s. Luhmann 1995a, 34f., 106f., 324ff.; s. a. Luhmann 1965, 176ff.). Auch in seiner ersten Bielefelder Vorlesung zu Interaktionssystemen aus dem Jahr 1969/ 70 diskutiert Luhmann zu Beginn den Gruppenbegriff und verwirft ihn als ein 
einem soziologisch weit verbreiteten Begriff: dem der sozialen Beziehung. Beim Beziehungsbegriff handele es sich, so Luhmann (1990a, 197, Fn. 2) um den »Ausweg aus einem schon verkorksten Theorieanfang«. Und in der Einleitung der Gesellschaft der Gesellschaft $(1997,24)$ heißt es knapp: die Annahme, dass die Gesellschaft aus Beziehungen zwischen Menschen bestehe, sei eine wesentliche »Erkenntnisblockierung() « der Gesellschaftstheorie. Erstaunlich ist bei diesen Einschätzungen weniger das Urteil Luhmanns an sich, sondern eher die Tatsache, dass er dabei den Beziehungsbegriff ausschließlich als einen Begriff im Rahmen der allgemeinen Theorie sozialer Systeme denkt. Es ist nun aber gerade nicht zwangsläufig, dass man dem Beziehungsbegriff einen solchen grundlagentheoretischen Stellenwert zusprechen muss.

In der Gesellschaft lassen sich vielmehr vielfältige Semantiken der Beziehung beobachten, die auf eine, um mit Georg Simmel zu formulieren, ganz spezifische Form der Vergesellschaftung fokussiert sind: Man spricht von Liebesoder Intimbeziehungen, von Familien- oderVerwandtschaftsbeziehungen, von Freundschafts- oder Bekanntschaftsbeziehungen. Dieser gesellschaftlichen Prominenz der Semantik der Beziehung, die doch offensichtlich auf einen besonderen Typ von Sozialität rekurriert, entspricht allerdings nicht nur in der Systemtheorie keine ausgearbeitete Soziologie der Beziehung selbst (vgl. nur Lenz 2006). So verwendet z.B. der sechsseitige Artikel Social relationships in adulthood in der 2001 neu aufgelegten International Encyplodia of the Social $\mathcal{E}$ Behavioral Sciences auf die Begriffsdefinition selbst kaum mehr als einen Halbsatz: »a relationship exists when people are at least minimally interdependent« (Clark 2001, 14423). ${ }^{2}$ Die Forschung zu sozialen Beziehungen ist wesentlich durch die Psychologie dominiert und war entsprechend zunächst dadurch gekennzeichnet, dass sie die individuellen Dispositionen der Beteiligten in den Vordergrund ihrer Modelle stellte und das Interdependenzverhältnis der Akteure wesentlich als wechselseitige Attraktivität konzipierte, so dass die Forschung häufig auf die (experimentell gut erfassbare) Initialinteraktion von Beziehungen konzentriert war. Mitte der 1970er Jahre entwickelte sich dann eine spezialisierte spersonal relationships<-Forschung (vgl. Perlman/Duck 2006), deren Verdienst es ist, dass sie nicht nur real bereits existierende Bezie-

soziologisch weiterführendes Konzept mit dem Argument, dass in der Literatur »die Gruppe letztlich doch immer aus Menschen bestehend gesehen «, der »Mensch als Teil der Gruppe« verstanden werde (Luhmann 1969/70, 9). In der neueren Organisationssoziologie Luhmanns (2000, 22ff.) findet sich nur noch eine marginale Bemerkung zur Gruppe, die auf die genannten systemtheoretischen Vorschläge keinen Bezug nimmt. Vielmehr notiert Luhmann am Ende des entsprechenden Abschnitts: »Inzwischen mehren sich die Anzeichen dafür, dass der Begriff der informalen Organisation und mit ihm der Gruppenbegriff durch eine Theorie der Interaktionssysteme ersetzt wird. (...) So kann man außerdem den theoretisch nicht sehr entwicklungsfähigen Gruppenbegriff durch die Theorie der Interaktion unter Anwesenden ersetzen.« (25) Ob es sich dabei wirklich um ein Ersetzungsverhältnis handeln kann, wird im Folgenden diskutiert.

2 Und vermischt dabei, ohne es zu bemerken, eine sozialtheoretische mit einer differenzierungstheoretischen Lesart des Begriffs. 
hungen in den Blick nahm, sondern damit auch die Aufmerksamkeit auf eine Abfolge von Interaktionen richtete, also auch auf das Bestands- bzw. Kontinuitätsproblem von Beziehungen. Im Rahmen dieser Forschung findet man dann die soziologisch interessante Feststellung, dass eine Beziehung eben nicht zureichend als Interdependenzverhältnis von Individuen zu begreifen sei, hinzutreten müsse vielmehr eine Bezugnahme der Interaktionen aufeinander (s. Hinde 1979, 14ff.). Man muss also zunächst zwischen Interaktion und Beziehung unterscheiden (können), um überhaupt von Beziehungen sprechen zu können. Einen entsprechend scharf gefassten Interaktionsbegriff bietet die Beziehungsforschung dann allerdings nicht - wohl aber die Systemtheorie.

\section{Der Beziehungsbegriff in Luhmanns Systemtheorie}

Luhmann hat den Beziehungsbegriff nicht immer schon so vehement abgelehnt wie oben zitiert. Vielmehr verwendet er ihn bis weit in die 1980er Jahre hinein in einer undogmatischen Weise. Zentral ist der Begriff der Beziehung in der Semantikstudie Liebe als Passion von 1982. Dort wird ohne Berührungsängste von »persönlicher « bzw. von »sozialer Beziehung « und »Intimbeziehung « gesprochen (1982, 13f.). Für den letzteren Fall, in der die individuellen Eigenschaften der beteiligten Personen wichtig werden, möchte Luhmann dann auch von »zwischenmenschlicher Interpenetration « sprechen. Den ursprünglich von Parsons stammenden Begriff der Interpenetration fasst Luhmann (1984, 289ff.) auf der Basis seiner Theorie operational geschlossenen Systeme so abstrakt, dass die These des wechselseitigen Zurverfügungstellens von Komplexität nicht nur auf das Verhältnis psychischer und sozialer Systeme begrenzt ist. Vielmehr unterscheidet Luhmann zwei Fälle von Interpenetration: soziale Interpenetration als das Verhältnis von Menschen und sozialen Systemen und zwischenmenschliche Interpenetration als das Verhältnis von Menschen (1984, 303ff., 317ff.). Auch wenn die zwischenmenschliche Interpenetration Verhältnisse des Inkommunikablen einschließt, »bleibt die Beziehung von Mensch zu Mensch [sic!] ein soziales Phänomen« $(1984,303)$, denn zwischenmenschliche Interpenetration kann nur über die Bildung eines sozialen Systems vermittelt werden. Mehr noch: die zwischenmenschliche Interpenetration konvergiert in der Luhmannschen Lesart dann eben mit der Intimbeziehung: »Wir wollen solche Beziehungen mit dem Begriff der zwischenmenschlichen Interpenetration kennzeichnen. Im gleichen Sinne kann man auch von Intimbeziehungen sprechen « (1982, 14, Hervorh. i.O.; s.a. 1982, 200; 1984, 305ff.). Die >Beziehung von Mensch zu Mensch - die zwischenmenschliche Interpenetration - wird also durch eine Beziehung - die Intimbeziehung - vermittelt.

Diese begriffliche Konfusion ist zunächst etwas irritierend, löst sich aber dann auf, wenn man eben die sozialtheoretische von der differenzierungstheoretischen Lesart des Begriffs unterscheidet. Luhmann selbst hat das in der Stu- 
die zu Intimbeziehungen (1982) wie auch in der entsprechenden Passage zum Interpenerationsbegriff in Soziale Systeme (1984) aber nicht dezidiert getan. Und nicht nur das: Gut 15 Jahre später will er für den empirisch ähnlich gelagerten Fall der Familie (1990) vom Beziehungsbegriff gar nichts mehr wissen. Das ist insofern überraschend, als man gerade mit Blick auf die familiäre Kommunikation vermuten könnte, dass es der Ausdifferenzierung eines spezifischen sozialen Systems bedarf, um zwischenmenschliche Interpenetration zu ermöglichen, die mit dem Begriff der Intimität selbst gerade noch nicht ausreichend bezeichnet ist. ${ }^{3}$ Statt dessen findet man die schon zitierte brüske und summarische Ablehnung des Beziehungsbegriffs: Jenseits der Systemtheorie werde mit >Beziehungen zwischen Menschen « etwas »ontologisch Minderwertiges« bezeichnet, das ihm vorausgehende Substanzen (Menschen) voraussetze; und in der Systemtheorie lenke der Begriff ab von der Tatsache, dass Sozialsysteme eben nicht Systeme >zwischen< Menschen seien, sondern Kommunikationssysteme, also Systeme bestenfalls >jenseits< von Menschen. Mit dieser Polemik gegen ein sozialtheoretisches Verständnis des Beziehungsbegriffs wird aber auch die Frage verdrängt, ob nicht gerade ein differenzierungstheoretisch verstandener Beziehungsbegriff eine distinkte Sozialform bezeichnet, deren angemessene Beschreibung der Systemtheorie bislang merkwürdig schwerfällt: Gemeint ist das paradoxe Phänomen einer Interaktion, die ihr eigenes Ende überdauert. Genau hier kommt der Beziehungsbegriff (wieder) ins Spiel, der nun aber kein Interdependenzverhältnis von Akteuren bezeichnet, sondern eben ein Interdependenzverhältnis von Interaktionen.

\section{Folgen der Umstellung auf den starken Interaktionsbegriff}

Die Interaktion als einfaches Sozialsystem (vgl. Luhmann 1969/70; 1975; 1997, 812ff.; ausf. Kieserling 1999) ist wesentlich dadurch bestimmt, dass die Kommunikation unter Anwesenden in dem Augenblick aufhört, in dem die Interaktionsteilnehmer auseinandergehen. Dies gilt selbst für den Fall, dass sich zu einem späteren Zeitpunkt dieselben Interaktionsteilnehmer wieder treffen. Auch wenn ein Beobachter einen engen Zusammenhang beider Zusammentreffen feststellen kann, so handelt es sich eben doch um zwei sich voneinander abgrenzende Sozialsysteme. Allerdings stehen diese Interaktionen offensichtlich in einem anderen Verhältnis zueinander, als es Interaktionen mit wechselnden Interaktionspartnern tun. Für diese Frage scheint der Luhmannsche

3 In Anlehnung an Simmel könnte man also sagen, dass Luhmann nicht ausreichend zwischen Form (Systemtyp) und Inhalt (Intimität) unterscheidet. Andererseits notiert er in Soziale Systeme: »Die Steigerung von Intimität ist durch eine funktionale Ausdifferenzierung entsprechender Kleinsysteme bedingt." (1984, 305; Hervorh. von mir) Dass damit nicht (nur) Interaktionssysteme gemeint sein können, legt schon das empirische Phänomen selbst nahe, das sich eben nicht in >one-night-stands< manifestiert. 
Interaktionsbegriff selbst aber zunächst nicht sensibel. Eine genaue Lektüre des Beitrags mit dem programmatischen Titel Einfache Sozialsysteme zeigt vielmehr, dass Luhmann (1975) sich im Anschluss an Goffman bei seinem Interaktionsverständnis stark an dem »Fall der elementaren Interaktion« orientiert: »der flüchtigen Begegnung, des kurzen Gesprächs zwischen Tür und Angel, der stummen gemeinsamen Fahrt im Eisenbahnabteil, des gemeinsam-ungeduldigen Wartens auf das Umschalten der Ampel« (1975, 21; Hervorh. von mir). Das sind die Fälle, die nach Luhmanns Einschätzung eine besondere Herausforderung für die Theorie sozialer Systeme darstellen, insofern es jeweils um Grenzfälle der Sozialität schlechthin geht. Genau besehen handelt es sich bei den Beispielen um das, was Erving Goffman mit snichtzentrierter Interaktion< bezeichnet hat - es geht in diesen Fällen gerade um das Vermeiden weitergehenden Sozialkontakts. Folge dieses Startpunktes ist die theoretisch zunächst attraktive minimale Bestimmung der Interaktion über Anwesenheit und wechselseitiges Wahrnehmen des Wahrnehmens, die Luhmann über Soziale Systeme bis hin zur Die Gesellschaft der Gesellschaft durchhält (vgl. 1984, 560ff.; 1997, 814ff.).Verfolgt man die Explikation des Interaktionsbegriffs im frühen Aufsatz zu Interaktion dann aber weiter $(1975,23 \mathrm{ff}$.), so wird deutlich, dass für - wieder mit Goffman gesprochen - >zentrierte Interaktionen munikation notwendig ist, um eine Abgrenzung gegenüber Situationen des reinen Wahrnehmens des Wahrnehmens zu ermöglichen. Um eine stabile (!) Ausdifferenzierung der Interaktion zu gewährleisten, sind vielmehr insbesondere die Themenorientierung der Interaktion und damit zusammenhängend die Ausbildung einer eigenen Systemgeschichte, d.h. die Strukturbildung der Interaktion von mindestens ebenso großer Wichtigkeit. Später heißt es dazu bei Luhmann in Soziale Systeme (1984, 564; Hervorh. von mir): »Bei genauerem Zusehen zeigt sich ..., daß autopoietische Erfordernisse der Weiterführung der Kommunikation Strukturbildungen erzwingen und daß man es daraufhin mit einer Differenz von Autopoiesis und Struktur zu tun hat. Die Strukturbildung wird vor allem dadurch erzwungen, daß Kommunikation von bloßer Wahrnehmung abgehoben werden muß ${ }^{4}{ }^{4}$ Mit den Kriterien der Anwesenheit und des wechselseitigen Wahrnehmens des Wahrnehmens werden also nur die Bedingungen der Möglichkeit einer Interaktion benannt; wie die Ausdifferenzierung der Interaktion dann aber genau erfolgt, ist damit gerade noch nicht bezeichnet. Ganz am Ende seiner Ausführungen in dem frühen Interaktionsaufsatz kommt Luhmann dann eher nebenbei und nur sehr knapp auch auf solche Interaktionssysteme zu sprechen, die durch ein Wiedersehen der Interaktionspartner gekennzeichnet sind. Dabei führt Luhmann einen neuen Begriff für Interaktionssysteme ein, die nicht mehr nur Situationssysteme sind: den der intermit-

4 Man findet eine entsprechende Überlegung aber auch schon in der dem Interaktionsaufsatz vorausgehenden Vorlesung zu einfachen Sozialsystemen. In dem Vorlesungsskript notiert Luhmann (1969/70, 18a): »Thematische Konzentration ermöglicht ... überhaupt erst eine gewisse autonome Selbststeuerung des Systems«. 
tierenden Systeme: »Intermittierende Systeme bleiben noch einfache Systeme ...; aber sie erfordern schon Abstraktionsleistungen, die das System auf eine neue Ebene struktureller Organisation bringen. (...) Nur intermittierend zusammentreffende Systeme entwickeln ein kommunikationsfähiges Wir-Bewußtsein, arbeiten eigene Verhaltensregeln aus und kontrollieren den Rekrutierungsprozess durch besondere Kommunikationen über die Zulassung neuer Mitglieder.« $(1975,32)$ Die Unschärfe der zitierten Textpassage ist nicht zufällig: >Intermittierende Systeme < bleiben noch >einfache Systeme<, aber bleiben sie auch Interaktionssysteme, wo doch für die Einzelinteraktion gerade konstitutiv ist, dass sie keine Kontinuität über die konkrete Interaktionssituation hinaus erreichen kann ${ }^{5}$ Was ist unter der >neuen Ebene struktureller Organisation< zu verstehen - gerade vor dem Hintergrund der aufgezeigten Bedeutung der Strukturbildung für die Ausdifferenzierung des Interaktionssystems? Die Hinweise auf die Ausbildung eines kommunikationsfähigen Wir-Bewusstseins, eigene Verhaltensregeln sowie die Kontrolle über den Rekrutierungsprozess von Mitgliedern, die stark in Richtung Organisation deuten, bleiben unausgearbeitet. ${ }^{6}$ Der Begriff der >intermittierenden Interaktion< selbst wird im weiteren Werk Luhmanns nicht mehr aufgegriffen.

Es scheint mir nun sinnvoller, auf die genannten Fragen nicht mit einer Ausweitung oder Modifikation des Interaktionsbegriffs zu antworten, sondern mit einem eigenständigen Begriff. Die umständliche und genau genommen fehlgehende Bezeichnung der sunterbrochenen Interaktion tische Problem, denn die konkrete Interaktion kann sich natürlich nicht unterbrechen und später wieder aufnehmen - sie ist dann eine neue Interaktion. Mit der Frage, ob der Begriff der intermittierenden Interaktion überhaupt sinnvoll ist, stößt man zunächst wieder auf den Gruppenbegriff, der ein ähnliches Phänomen im Blick zu haben scheint: So steht im Zentrum der Überlegungen zu einem systemtheoretischen Gruppenbegriff bei Hartmann Tyrell (1983) insbesondere die Abgrenzung von der Interaktion einerseits und der Organisation andererseits: Gruppen sind im Unterschied zu Organisationen zwar interaktionsbasiert, gleichzeitig kann die Interaktionsbasiertheit aber nicht das entscheidende Definitionsmerkmal der Gruppe sein, da sie die Einzelinteraktionen eben gerade überdauert. Die für den systemtheoretischen Zugang konstitutive Frage, was die Einheit der Gruppe ausmacht, wird von Tyrell dann mit der Doppelformel »Zugehörigkeit« bzw. »Zusammengehörigkeit« beantwortet (1983, 81). Damit ist aber eher ein Problem markiert als eine Lösung formuliert. Dies wird schon an der Begriffsdopplung deutlich, die einerseits auf ein Verhältnis

5 Auch hier lohnt ein Blick in das Vorlesungsskript: Luhmann spricht dort das »Sonderproblem der Systemkontinuität trotz Unterbrechung « an und betont, dass der Begriff des einfachen Sozialsystems nicht nur »Situationssysteme im engeren Sinne von nichtunterbrochenen Einheiten« umfasst $(1969 / 70,20)$.

6 Allein in einer Fußnote findet sich ein bezeichnender Hinweis: »Hier liegt dann auch der Übergang zu dem, was die ältere Soziologie unter dem Begriff der primary group erörtert hat.« (Luhmann 1975, 36, Fn. 41) 
von Personen zu einem Sozialsystem - >Zugehörigkeit<-, andererseits von Personen zueinander - >Zusammengehörigkeit $<$ - abstellt. Mit letzterem wird zudem die Bestimmung der Grenze des Sozialsystems in dessen Umwelt verlegt: Das Sozialsystem Gruppe wird durch ein Phänomen konstituiert, das jenseits seiner sozialen >Materialisierung angesiedelt ist. Man sieht zunächst also den Verdacht Luhmanns gegen ein Konzept bestätigt, das offensichtlich aus einer auf dem Kommunikationsbegriff basierenden Soziologie hinausführt.

Trotzdem gilt es festzuhalten, dass mit der Abgrenzung zum Interaktionsbegriff durch die zunächst etwas unscharfe Formulierung der Dauerhaftigkeit genau das Problem benannt ist, das >unterbrochene Interaktionen haben: Sie müssen die Latenzphase zwischen den einzelnen Interaktionen überbrücken. Was aber ist dabei überhaupt latent? Wird die Interaktion beendet, ist sie selbst dann nicht latent vorhanden, wenn ein weiteres Zusammentreffen absehbar ist: Erst die >Unterbrechungく der Interaktion ermöglicht überhaupt die >Kontinuierung der Interaktion. Die These der Kontinuität der Interaktion kann sich also nicht auf die Operationsebene selbst beziehen, sondern allenfalls auf die Ebene der Struktur der Interaktion: Obwohl die Interaktion beendet wird, kann eine nachfolgende Interaktion auf die Strukturen zurückgreifen, die in einer vorhergehenden Interaktion entwickelt worden sind und insofern direkt an diese anschließen. Dieser Strukturtransfer ist aber nur dann möglich, wenn eine Zuordnung der beiden Interaktionen zueinander auch hergestellt werden kann. Es muss also so etwas wie eine Identität einer Mehrzahl von Interaktionen geben, die es erlaubt, ganz bestimmte Interaktionen aufeinander zu beziehen und dadurch eine >Kontinuierung< der Interaktion sicherzustellen, bei der die bisherige Interaktionsgeschichte auch für die neue Interaktion Strukturwirkung hat. Die Interaktion kann dann auch beendet werden in der Erwartung, dass, um es paradox zu formulieren, das Ende der Interaktion nicht das Ende der Interaktion ist. Wie aber wird die Einheit einer solchen Iteration von Interaktionen sichergestellt?

\section{IV. >Beziehung< als Selbstbeschreibung eines Interaktionszusammenhangs}

Um diese Frage zu beantworten, möchte ich zunächst an einen Vorschlag André Kieserlings anknüpfen und den Begriff des Interaktionszusammenhangs einführen. Der Begriff bezeichnet »eine Mehrheit von Interaktionssystemen ..., sofern sie einheitlich erwartet und einheitlich thematisiert werden kann. (...) In der Form der Erwartbarkeit solcher Erwartungen wirkt der Interaktionszusammenhang an der Selbststeuerung der Einzelinteraktionen mit« $(1999,221)$. Der Interaktionszusammenhang stellt einen Horizont für die Einzelinteraktionen dar: Die Interaktion versteht sich selbst als eine Episode des Interaktionszusammenhangs, die zwischen vergangenen und zukünftigen Interaktionen 
vermittelt. Daraus resultiert eine Doppelorientierung der einzelnen Kommunikation, die nicht nur die unmittelbare Anschlussfähigkeit in der Interaktion im Blick hat, sondern auch den Interaktionszusammenhang selbst, ohne dass diese Orientierung auf den Interaktionszusammenhang die Autonomie der jeweiligen Interaktion in Frage stellt. Durch die Einbettung in einen Interaktionszusammenhang kommt also zu einer ganz spezifischen, nämlich strukturellen Entgrenzung der Einzelinteraktion, ohne dass sie operativ ihre Grenzen überschreitet.

Der Begriff des Interaktionszusammenhangs ist zunächst so allgemein formuliert, dass er dazu befähigt, verschiedene empirische Phänomene unter der hier interessierenden Fragestellung zu betrachten. Er spezifiziert aber gerade noch nicht, wie es möglich, dass sich bestimmte Interaktionen aufeinander beziehen und so ein Zusammenhang von Interaktionen, der über die Einzelinteraktion hinausreicht, konstituiert wird. Stellt man diese Frage ins Zentrum, so lassen sich zwei grundsätzlich unterschiedliche Möglichkeiten der Generierung eines Zusammenhangs von Interaktionen unterscheiden: In dem einen Fall ist die Konstitution des Interaktionszusammenhangs von Strukturvorgaben seiner Umwelt abhängig, in dem anderen Fall erzeugt er die Strukturen, die die Einheit mehrerer Interaktionen garantieren, selbst. ${ }^{7}$

1) Die Strukturierung einer Interaktion läuft im wesentlich über die eigene Systemgeschichte: Was in der Interaktion bereits passiert ist, limitiert in vielerlei Hinsicht das, was danach noch möglich ist. Eine besondere Rolle für die Selbststeuerung der Interaktion spielt dabei das Thema der Interaktion, das man deshalb als das wesentliche Strukturmuster der Interaktion verstehen kann (vgl. Luhmann 1975, 23ff.; Kieserling 1999, 180ff.): Das Thema ermöglicht die Auswahl von Beiträgen und stellt so erst die Anschlussfähigkeit von Kommunikationen und damit die Ausdifferenzierung einer Interaktion sicher, indem es bestimmte Beiträge - eben solche zum Thema - wahrscheinlicher macht. Nun ist aber schon innerhalb einer Interaktion ein Themenwechsel möglich, ohne dass die Interaktion beendet wird; das Thema selbst ist also in der Regel eine zu schwache Struktur, um die Einheit einer Interaktion, geschweige denn die Einheit eines Zusammenhangs verschiedener Interaktionen selbst garantieren zu können. Deshalb kann man beobachten, dass die Konstitution eines Interaktionszusammenhangs, der durch ein kontinuierliches Interesse an einem Thema (oder zumindest einem Themenbereich) gekennzeichnet ist, in der Regel von Strukturvorgaben aus der Umwelt des Systems abhängig ist. Häufig handelt es sich dabei um Strukturen eines anderen sozialen Systems, ${ }^{8}$ in der

7 Es handelt sich dabei nicht um sich wechselseitig ausschließende Problemlösungen. Fragen des Wiedereintritts der Unterscheidung in sich selbst, wie sie z.B. bei Geschäftsbeziehungen oder informellen Beziehungen in Organisationen vorliegt, können hier aber aus Platzgründen nicht diskutiert werden.

8 Das ist aber nicht zwingend: auch eine räumliche (Vor)Strukturierung der Interaktionssituation, die die Interaktionswiederaufnahme erleichtert und dabei zugleich häufig auch eine gewisse Themenpräferenz vorgibt, ist als eine solche externe Strukturvorgabe beobachtbar 
modernen Gesellschaft insbesondere um die einer Organisation. Auch wenn man Organisationen nicht zureichend als eine Anhäufung von Interaktionen beschreiben kann (s. Luhmann 2000), finden innerhalb von Organisationen doch eine Vielzahl von Interaktionen statt, die nicht in Einzelinteraktionen aufgehen: Abteilungen, Teams, Stäbe, Arbeitsgruppen - alle diese internen Differenzierungsformen einer Organisation sind nicht nur durch eine Interaktionsverdichtung, sondern auch durch eine Interaktionskontinuität gekennzeichnet. Dabei stellt die Organisation zunächst über ihr systemkonstitutives Kriterium der Mitgliedschaft sicher, dass in einer Interaktion davon ausgegangen werden kann, dass dieselben Interaktionspartner wieder zusammentreffen werden, um das Thema und damit die Interaktion fortzusetzen: Gerade in der und durch die Organisation gilt das »Gesetz des Wiedersehens« (Luhmann 1965, 170). Zugleich regeln die Entscheidungsprogramme und die Stellenstruktur der Organisation, welche Themen eine Interaktion behandelt und durch eine entsprechende Verteilung der Sprecherrollen, in welcher Form die Themenbehandlung abläuft. Dazu gehört dann auch eine mögliche Kontinuierung der Interaktion, wobei der Zusammenhang mehrerer Interaktionen, also die zeitliche Kontinuität trotz Unterbrechung der Kommunikation, in sachlicher wie sozialer Hinsicht durch die Struktur der Organisation garantiert wird. Die Interaktion delegiert die Lösung ihres Problems der `Fortsetzung` an die Organisation, indem sie sich in ihrem Operieren als Interaktion von den Strukturvorgaben der Organisation abhängig macht, statt sich nur auf die eigene Systemgeschichte zu verlassen. Insofern kann man von einem organisierten Interaktionszusammenhang sprechen, der eben gerade kein selbstorganisierter Interaktionszusammenhang ist: Die Einheit mehrerer Interaktionen wird erst durch die Organisationsstruktur sichergestellt, ohne dass die Organisation selbst vollständig kontrollieren könnte, wie die einzelnen Interaktionen ablaufen, da die Autonomie der Interaktionen trotz der strukturellen Kopplung an die Organisation selbst nicht in Frage gestellt wird.

2) Interaktionen müssen aber zu ihrer Kontinuierung nicht zwingend auf die Strukturvorgaben anderer Sozialsysteme zurückgreifen, vielmehr gibt es auch eine selbstgenerierte, wenn man so will: sinteraktionsinterne< Lösung, die allein mit den Möglichkeiten auskommt, die die Interaktion selbst bietet. Die hier vertretene These ist, dass eine solche autonome Konstitution eines Zusammenhangs mehrerer Interaktionen durch eine spezifische Form der Selbstbeobachtung des Interaktionszusammenhangs erreicht wird. Diese Selbstbeobachtung findet in der Beziehungssemantik ihren Ausdruck, die die Einheit des Interaktionszusammenhangs durch die Orientierung an den beteiligten Interaktionspartnern herstellt. Die Einheitsbeschreibung greift dabei auf ein interaktionsspezifisches Merkmal zurück - und deshalb fällt es der Soziologie vielleicht

(s. Feld 1981). Häufig wird man dabei aber feststellen, dass auch hier Organisationen wieder relevant werden. 
auch bisweilen so schwer, zwischen Interaktion und Beziehung zu unterscheiden -, indem sie nämlich darauf reagiert, dass sich in Interaktionen Personen aufdrängen. Personen sind zunächst kommunikative Zuschreibungen, sie sind Folge von Zurechnungsnotwendigkeiten in der Kommunikation: Die Kommunikation beobachtet sich als Handlung und schreibt das Mitteilungshandeln einer Person zu. Die Person wird so zu einem kommunikativen Artefakt in Form spezifischer »Erwartungskollagen, die im System als Bezugspunkte für weitere Selektionen fungieren « (Luhmann 1984, 178). Durch die mitteilungsbezogene Selbstsimplifizierung der Kommunikation als Handlung und die Zentralstellung der wechselseitigen Wahrnehmung der Interaktionsteilnehmer ist eine solche Personalisierung in der Interaktion nahezu unvermeidbar. Die Person dient aber darüber hinaus auch als eine »Aufzeichnungsstelle() für ... sequentielle Kommunikationsverläufe« (Luhmann 1990b, 34), sie ist also per se eine besondere Form der Beobachtung des Zusammenhangs mehrerer Situationen (Luhmann 2000, 285). So wie die Abfolge mehrerer Interaktionen es (erst) ermöglicht, die Person als etwas über die einzelne Interaktionen hinaus Konstantes kennenzulernen, so ermöglicht umgekehrt die (kommunikativ unterstellte) Konstanz der an den Interaktionen beteiligten Personen eine Einheitsbeobachtung einer Mehrzahl von Interaktionen. Die Einheitsbeschreibung eines Zusammenhangs verschiedener Interaktionen kann sich deshalb an der Identität dieser Personen orientieren, es handelt sich um einen in den Personen der Interaktionsteilnehmer sverankerten< Interaktionszusammenhang, um eine Terminologie Erving Goffmans (1982, 257f.) aufzunehmen: Die Unabhängigkeit der Konstitution des Interaktionszusammenhangs von Strukturvorgaben seiner Umwelt kommt dann in der Selbstbeschreibung als Wechselverhältnis der beteiligten Interaktionspartner zum Ausdruck.

Die Selbstbeobachtung als Beziehung stellt also ein wesentliches Moment in der Konstitution des Zusammenhangs von Interaktionen dar. Dabei muss es sich nicht unbedingt um eine reflexive Kommunikation über die Identität des Interaktionszusammenhangs handeln, vielmehr reicht für den alltäglichen Betrieb einer Beziehung in der Regel eine Symbolisierung der Einheit des Interaktionszusammenhangs völlig aus. Dies geschieht entweder durch eine Beziehungsbezeichnung wie Freundschaft, Ehe, Familie bzw. die entsprechende Bezeichnung der Beziehungsbeteiligten, aber auch durch operativ verwendbare Kurzformel wie z.B. die des >Wir< (s.a. Luhmann 1984, 618f.) oder anderer, auch äußerlich wahrnehmbarer »Beziehungszeichen « (Goffman 1982, 262ff.), die die Einheit des Interaktionszusammenhangs markieren, wie z.B. beziehungsspezifische Formen des Begrüßens oder des Körperverhaltens. Solche operativ verwendbaren Einheitssymbole wirken zugleich als ein Motiv zur Interaktionsteilnahme, erzeugen also ein Bindungseffekt des Interaktionszusammenhangs gegenüber seinen Teilnehmern; darauf rekurriert wohl letztlich auch der Tyrellsche Gruppenbegriff mit seinem Doppelkriterium von >Zugehörigkeit und `Zusammengehörigkeit . Im kommunikationstheoretischen 
Verständnis des Interaktionszusammenhangs handelt es sich dabei aber um einen Effekt des interaktiven Geschehens, der sich mittels der Form >Person< (Luhmann 1995b) den strukturell an die Interaktion gekoppelten psychischen Systemen mitteilt, und nicht um ein (dann sowieso nur psychisches) Phänomen, das den Zusammenhang der Interaktionen selbst begründet.

In lockerer Anlehnung an eine Diskussion über das Autopoiesiskonzept bei Gunther Teubner (1987) kann man mit Blick auf den so konstituierten Interaktionszusammenhang von einem autopoietischen System zweiter Ordnung sprechen, dessen Besonderheit darin liegt, dass es sich auf der Basis anderer autopoietischer Systeme ausdifferenziert und diese dann in einer spezifischen Weise reintegriert, indem sie sie aufeinander bezieht. ${ }^{9}$ Dadurch wird nicht nur die nach wie vor bestehende Autonomie der Interaktionen gegenüber sihrem< Interaktionszusammenhang betont, sondern umgekehrt auch deutlich, dass die Interaktionen selbst durch ihre Einbettung in einen Interaktionszusammenhang wiederum zu emergenten Systemeinheiten werden, was nicht ohne Folgen für die jeweils möglichen Kommunikationen in diesen Interaktionen bleibt: In solchen Interaktionen wird anders kommuniziert als in Situationen, die keine gemeinsame vergangene und / oder zukünftige Interaktionsgeschichte haben. Dabei erzeugt der Interaktionszusammenhang die eigenen Struktur selbst, indem es über die Beobachtung des Zusammenhangs von Interaktionen zu einer Systemgeschichte der präsent gehaltenen Selektionsleistungen vergangener Interaktionen kommt, die dann auch zukünftige Interaktionen instruiert sowie zwischen zum Interaktionszusammenhang gehörenden und anderen Interaktionen zu unterscheiden erlaubt. Erst die Selbstbeschreibung des Interaktionszusammenhangs als Beziehung ermöglicht eine Zuordnung von Interaktionen zueinander und damit einen legitimen Strukturtransfer von einer Interaktion zur nächsten, so dass die Kommunikation die Systemgrenze der Interaktion transzendiert, ohne dass die Differenz zwischen den Interaktionen aufgehoben wird. Die für den Gruppenbegriff wie für den Begriff des intermittierenden Systems lästige Frage nach dem Bestand >zwischen < der Materialisierung in den einzelnen Interaktionen und auch die problematische These der >relativen Dauerhaftigkeit< als Systemcharakteristikum entfällt damit: Die Beziehung >bestehtı, solange über die entsprechende Selbstbeschreibung eine Zuordnung von Interaktionen zueinander erfolgt.

9 Dagegen betont André Kieserling (1999, 223; Hervorh.i. O.), »daß der Interaktionszusammenhang selbst kein Interaktionssystem ist, sondern eine Struktur dieses und anderer Sozialsysteme darstellt. « Dass es sich bei dem sich als >Beziehung`selbstbeschreibenden Interaktionszusammenhang nicht um eine (Einzel-)Interaktion handelt, wird hier natürlich nicht bestritten. Die interessantere Frage ist aber, ob man nicht von einer Autonomie des Interaktionszusammenhangs ausgehen muss, die in der Selbstorganisation seiner Strukturen begründet liegt. Indem Kieserling vor dem Hintergrund des sharten ^Autopoiesistheorems die Möglichkeit eines Systemstatus' von vornherein ausschließt, begibt er sich aber auch der Möglichkeit, zwischen fremd- und selbstorganisierten Interaktionszusammenhängen zu unterscheiden. 


\section{Literatur}

Clark, M.S. (2001): Social relationships in adulthood. International Encyclopedia of the Social and Behavioral Sciences, 14423-14429.

Feld, Scott L. (1981): The focused organization of social ties. American Journal of Sociology 86, 1015-1035.

Goffman, Erving (1982): Das Individuum im öffentlichen Austausch. Mikrostudien zur öffentlichen Ordnung. Frankfurt a.M.: Suhrkamp.

Hinde, Robert (1979): Towards Unterstanding Social Relationships. London: Academic Press.

Kieserling, André (1999): Kommunikation unter Anwesenden. Studien über Interaktionssysteme. Frankfurt a.M.: Suhrkamp.

Lenz, Karl (2006): Soziologie der Zweierbeziehung. Eine Einführung. 3. überarb. Aufl. Opladen:VS.

Luhmann, Niklas (1965): Spontane Ordnungsbildung. S. 163-183 in: F. Morstein Marx (Hg.), Verwaltung. Eine einführende Darstellung. Berlin: Duncker \& Humblot.

Luhmann, Niklas (1969/70): Einfache Sozialsysteme. Unveröff. Vorlesungsskript WS $1969 / 70$. Bielefeld.

Luhmann, Niklas (1975): Einfache Sozialsysteme [1972]. S. 21-38 in: Ders., Soziologische Aufklärung 2: Aufsätze zur Theorie der Gesellschaft. Opladen: Westdt.Verlag.

Luhmann, Niklas (1982): Liebe als Passion. Zur Codierung von Intimität. Frankfurt a.M.: Suhrkamp.

Luhmann, Niklas (1984): Soziale Systeme. Grundriß einer allgemeinen Theorie. Frankfurt a.M.: Suhrkamp.

Luhmann, Niklas (1990a): Sozialsystem Familie. S. 196-217 in: Ders., Soziologische Aufklärung 5: Konstruktivistische Perspektiven. Opladen. Westdt. Verlag.

Luhmann, Niklas (1990b): Die Wissenschaft der Gesellschaft. Frankfurt a.M.: Suhrkamp.

Luhmann, Niklas (1995a): Funktionen und Folgen formaler Organisation. Mit einem Epilog 1994. 4. Aufl. Berlin: Duncker \& Humblot.

Luhmann, Niklas (1995b): Die Form »Person«. S. 142-154 in: Ders., Soziologische Aufklärung 6: Die Soziologie und der Mensch. Opladen: Westdt.Verlag.

Luhmann, Niklas (1997): Die Gesellschaft der Gesellschaft. Frankfurt a.M.: Suhrkamp.

Luhmann, Niklas (2000): Organisation und Entscheidung. Opladen: Westdt. Verlag.

Neidhardt, Friedhelm (1979): Das innere System sozialer Gruppen. Kölner Zeitschrift für Soziologie und Sozialpsychologie 31, 639-660.

Perlman, Daniel / Duck, Steve (2006): The seven seas of the study of personal relationships: From »the thousand islands « to interconnected waterways. S. 3-34 in: A.L. Vangelisti / D. Perlman (eds.), The Cambridge Handbook of Personal Relationships. Cambridge: Cambridge UP.

Teubner, Gunther (1987): Episodenverknüpfung. Zur Steigerung von Selbstreferenz im Recht. S. 423-446 in: D. Baecker et al, Theorie als Passion. Niklas Luhmann zum 60. Geburtstag. Frankfurt a.M.: Suhrkamp.

Tyrell, Hartmann (1983): Zwischen Interaktion und Organisation I: Gruppe als Systemtyp. S. 75-87 in: F. Neidhardt (Hg.), Gruppensoziologie. Perspektiven und Materialien. Sonderheft 25 der KZfSS. Opladen: Westdt.Verlag.

Willke, Helmut (1978): Elemente einer Systemtheorie der Gruppe: Umweltbezug und Prozesssteuerung. Soziale Welt 29, 243-257.

Dipl.-Soz. Johannes F.K. Schmidt

Soziologisches Seminar, Universität Luzern

Postfach 7455, CH-6000 Luzern 7

johannes.schmidt@unilu.ch 